Document downloaded from:

http://hdl.handle.net/10251/104265

This paper must be cited as:

Morillo-Torres, D.; Barber, F.; Salido, MA. (2017). A new model and metaheuristic approach for the energy-based resource-constrained scheduling problem. Proceedings of the Institution of Mechanical Engineers Part B Journal of Engineering Manufacture. 1(1):1-13. doi:10.1177/0954405417711734

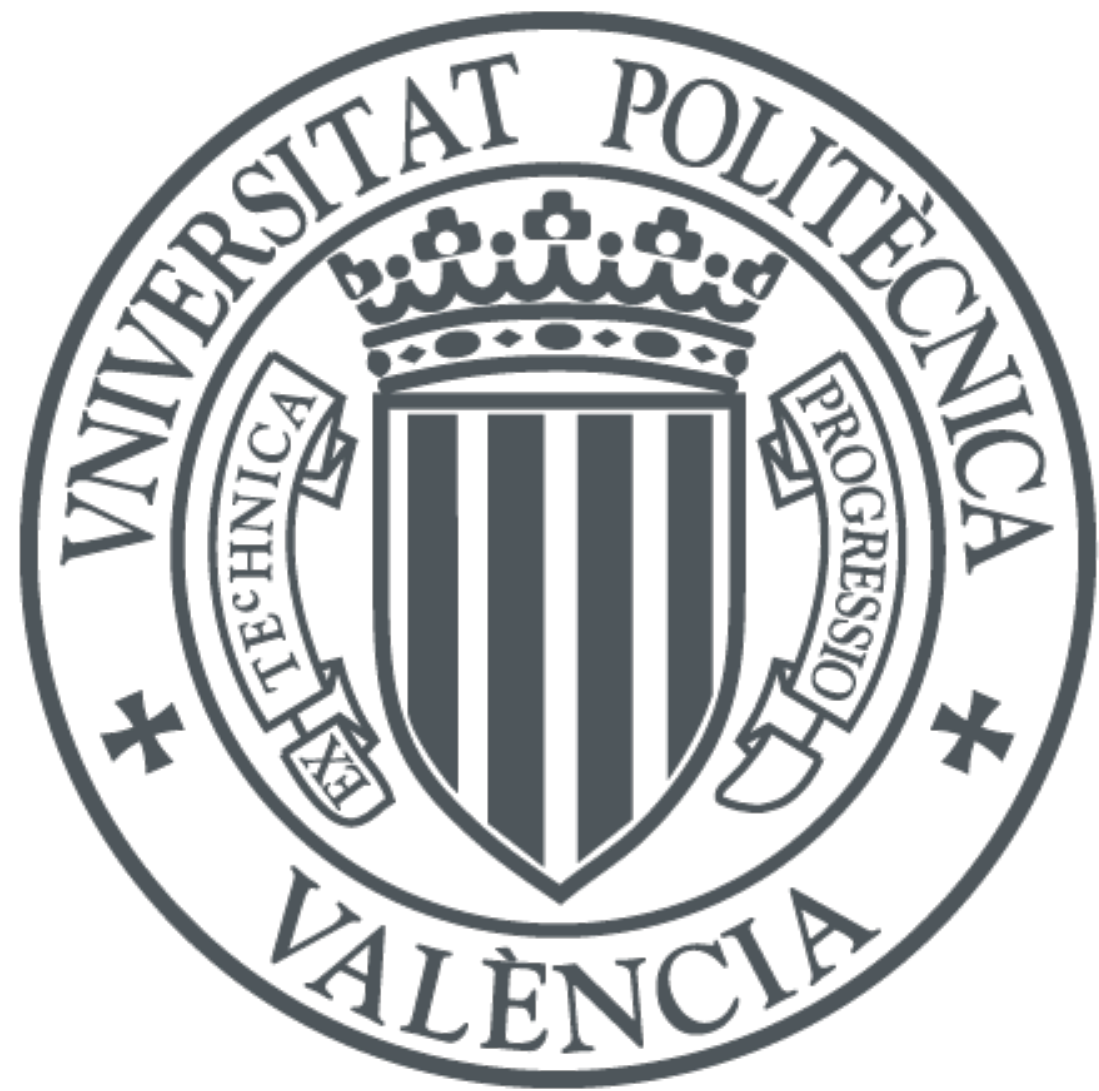

The final publication is available at

https://doi.org/10.1177/0954405417711734

Copyright SAGE Publications

Additional Information 


\title{
A new model and metaheuristic approach for the energy-based
}

Journal Title

$\mathrm{XX}(\mathrm{X}): 2-28$

(C) The Author(s) 2015

Reprints and permission:

sagepub.co.uk/journalsPermissions.nav DOI: $10.1177 /$ ToBeAssigned

www.sagepub.com/

(S)SAGE

\section{resource-constrained scheduling problem}

\section{Daniel Morillo Torres, Federico Barber and Miguel A. Salido}

\begin{abstract}
This paper focuses on obtaining sustainable and energy-efficient solutions for limited resource programming problems. To this end, a model for integrating makespan and energy consumption objectives in multi-mode resource-constrained project scheduling problems (MRCPSP-ENERGY) is proposed. In addition, a metaheuristic approach for the efficient resolution of these problems is developed. In order to assess the appropriateness of theses proposals, the well-known Project Scheduling Problem Library (PSPLIB) is extended (called PSPLIB-ENERGY) to include energy consumption to each RCPSP (Resource-Constrained Project Scheduling Problem) instance, through a realistic mathematical model. This extension provides an alternative to the current trend of numerous researches about optimization and the manufacturing field, which require the inclusion of components to reduce the environmental impact in the decision-making process. PSPLIB-ENERGY is available at http://gps. webs .upv.es/psplib-energy/.
\end{abstract}

\section{Keywords}

MRCPSP, PSPLIB, energy consumption, sustainable scheduling, metaheuristic, Genetic Algorithms

Prepared using sagej.cls [Version: 2015/06/09 v1.01] 


\section{Introduction}

One of the main challenges in industry is to optimally carry out the process of decision making. The current trend in various academic areas is oriented towards environmental awareness [1, 2, 3]. More precisely, in the last decade, eco-efficiency solutions have taken a relevant position in firms due to pressures from government regulators, community activists, global competition, and nongovernmental organizations [4]. As a consequence, the need for measuring the impact of sustainable solutions has increased since then [5].

In the artificial intelligence field, as well as in operations research, there is a subset of characteristic problems called scheduling problems, whose objective is to properly allocate available resources to the activities in order to optimize an objective function that is usually related to time (e.g., the total execution time of the project (makespan), tardiness, maximum lateness, etc.) [6]. Resources can be machines, materials, people, money, time, etc. These problems have great importance in industry due to their applications in different fields such as production, distribution, transportation, project management, and supply-chain optimization in general. Currently, the research effort is focused on analyzing and developing new methodologies for the optimal allocation of resources to minimize both makespan and energy consumption [7, 8].

In general, scheduling problems are combinatorial optimization problems. Therefore, relatively small instances are highly complex, with most of them being categorized as NP-hard, such as the job shop scheduling problem (JSP), the flow shop scheduling problem (FSP), and the multi-mode resource-constrained project scheduling problem (MRCPSP). Therefore, due to inherent complexity, it is not possible to find an optimal solution in a reasonable period of time. However, these problems show great applicability to real world situations. Hence, the research community is constantly developing and improving the techniques

\footnotetext{
${ }^{1}$ Instituto de Automática e Informática Industrial, Universitat Politècnica de València 
and methods to solve these problems. Similarly, benchmark libraries are needed to compare and evaluate these algorithms in an empirical way.

The MRCPSP is considered to be one of the most important scheduling problems [9]. All benchmarks and most algorithms than have been developed to solve this problem have focused on minimizing makespan. However, there are few studies about optimizing energy consumption in MRCPSP.

There are two main contributions of this paper. The first one is to propose a specific resource-constrained scheduling problem that takes into account both energy consumption and makespan in order to optimize the project in an efficient way. The second one is to provide a test instance library to compare different algorithms to solve the proposed problem.

Thus, this work is focused on analyzing the MRCPSP in order to propose MRCPSP-ENERGY, which is a single-objective problem where the activities have different energy consumptions to be executed at different rates. The objective is to minimize both makespan and energy consumption. Furthermore, an extension of the most commonly used library to solve the MRCPSP, the PSPLIB library (Project Scheduling Problem Library) [10], is proposed. To this end, a mathematical model to relate the energy consumption and the processing time of activities is proposed. Thereby, the instances of the PSPLIB library are extended by associating an energy consumption to each activity of the RCPSP. This new library, called PSPLIB-ENERGY, is available at http: //gps.webs.upv.es/psplib-energy/. Finally, a genetic algorithm for solving these instances is proposed to compare the performance of new algorithms. It is based on the heuristic methodologies that produce the best results for solving the MRCPSP.

\section{Literature review}

There are different strategies for energy optimization in manufacturing processes. These strategies can be classified into two levels: the machine tool level and manufacturing system level [11]. The first level refers to improving the process taking into account the machine design that performs the operation from a technological point of view [12, 13, 14]. For a comprehensive review about this first level, we refer the reader to [15]. The second level emphasizes energy optimization through the management and allocation of the activities and resources with the objective of minimizing energy requirements [16, 1, 17]. Energy optimization at this level is of great importance and provides several

Prepared using sagej.cls 
improvement opportunities [18, 19]. This work focuses on this second level to minimize makespan and energy consumption as a single-objective function.

For the manufacturing system level, there are several studies that deal with scheduling optimization taking into account energy consumption, both from a general point of view, by analyzing general scheduling problems as well as from a specific perspective by applying them to specific manufacturing areas. Among these research studies, one of the most important ones was proposed by Mouzon et al. [1]. They developed a multi-objective model for minimizing both the energy consumption and makespan of a single machine scheduling problem. They found that it is possible to save up to $80 \%$ of energy when non-bottleneck machines were turned off until needed during the period of next job arrival. Fang et al. [20] developed a new approach to schedule a flow shop problem in manufacturing for power consumption and carbon footprint reduction. Bruzzone et al. [16] provided an energy-aware mathematical model for the flexible flow shop problem to modify a given schedule in order to account for peaks of power. Artigues et al. [21] analyzed an industrial case-study, which led to a generic problem called the Energy Scheduling Problem. It consists of scheduling a set of activities which have a required total energy and have to be processed using a constrained energy resource. The energy amount used by an activity can be different for each period of time. Activities have a release date and deadline. Furthermore, they have a minimum and maximum energy consumption. The goal is to find the start time of each activity. Luo et al. [22] proposed a new ant colony optimization for solving a hybrid flow shop scheduling problem taking into account machine electricity consumption cost.

More recently, Okubo et al. [23] proposed a model that can deal with energy consumption during the setup operation on the RCPSP by using partially renewable resources. Zhang et al. [24] proposed a mathematical formulation for minimizing the energy consumption of a machining system by integrating planning and scheduling. The solution method was a genetic algorithm. That model considers a set of jobs with few alternative process plans and a set of machines. Each job is loaded and processed according to the predetermined sequence of operations in the process plan. Thus, the objective is to find the optimal process plan and machine allocation for each job. Li et al. [25] proposed a multi-objective mathematical model for the permutation flow line scheduling problem in order to simultaneously minimize the total flow-time and the energy consumption. Since this problem has a NP-hard complexity, they adapted the non-dominated sorting genetic algorithm II to solve it.

Prepared using sagej.cls 
However, there are few studies that consider energy efficiency or provide a common benchmark where these methods can be evaluated. Specifically, the MRCPSP under energy efficiency optimization has not been studied in great depth, and there is not a common benchmark that includes both makespan and energy consumption. Therefore, the library proposed in this paper is a clear contribution to the evaluation of energy efficiency-based solutions to the MRCPSP.

On the other hand, different metaheuristic strategies to solve the MRCPSP have been proposed. These are mainly based on genetic algorithms, scatter search, simulated annealing, particle swarm optimization, among others. According to computational experiments from academic literature, the population-based heuristics are those that achieve the best results. For an extensive review of the most relevant methodologies, we refer the reader to $[26,27]$.

\section{Description of the MRCPSP}

Formally, the standard MRCPSP can be defined as follows [28]. A project consists of a set $I$ of $n$ activities $I=\{1, \ldots, i, \ldots, n\}$, a set $B$ of $K^{\rho}$ shared renewable resources $B=\left\{1, \ldots, b, \ldots K^{\rho}\right\}$, and there is a maximum amount $R_{b}^{\rho}$ of every renewable resource. There exists a set $K$ of $K^{\nu}$ shared non-renewable resources $K=\left\{1, \ldots, k, \ldots K^{\nu}\right\}$, and there is a maximum amount $R_{k}^{\nu}$ of every non-renewable resource. A list of all the symbols used in this paper is included in the Appendix.

Each activity $i \in I$ has $m=\{1, \ldots, M\}$ execution modes and a non-preemptive execution time $d_{i m}$, which requires a total $r_{i m b}^{\rho}$ renewable resource of each type $b$ and a total $r_{i m k}^{\nu}$ non-renewable resource of each type $k$ for its realization. Generally activities 1 and $n$ are dummy activities and their duration and resource consumption are zero, representing the start and end of the project. Activities are subject to precedence constraints, which indicate that each activity cannot be started before all its predecessor activities are completed. The objective is to minimize the total duration of the project.

The MRCPSP is a well-known NP-hard problem [29]. It can be modeled as a mixed integer linear programming formulation which is detailed in Expressions (1) to (6). The decision variables $\xi_{\text {imt }}$ take the value one when the activity $i$ is executed in mode $m$ and finishes at time $t$, and zero otherwise. It is noteworthy that this formulation needs time intervals: an early start time and a late start

Prepared using sagej.cls 
time $\left[e s_{i}, l s_{i}\right]$ for each activity by computing an upper bound and applying the method of forward pass and backward pass with the lowest execution time of activities.

Expression (1) shows the optimization function where the objective is to minimize the finish time of the last activity $(n)$. Expression (2) ensures that each activity starts only once. Expression (3) represents the precedence constraints, and the set $P_{i}$ contains the immediate predecessor activities of activity $i$. Expressions (4) and (5) ensure that the capacity of each type of resource is not exceeded.

$$
\operatorname{Min}: \sum_{t=e s_{n}}^{l s_{n}} t * \xi_{n 1 t}
$$

Subject to:

$$
\begin{gathered}
\sum_{m=1}^{M} \sum_{t=e s_{i}}^{l s_{i}} \xi_{i m t}=1 \quad \forall i \in I \\
\sum_{m=1}^{M} \sum_{t=e s_{j}}^{l s_{j}} t * \xi_{j m t} \leq \sum_{m=1}^{M} \sum_{t=e s_{i}}^{l s_{i}}\left(t-d_{i m}\right) * \xi_{i m t} \quad \forall j \in P_{i}, \forall i \in I \\
\sum_{i=0}^{n} \sum_{m=1}^{M} r_{i m b}^{\rho} * \sum_{s=m a x\left\{t-d_{i m}, e s_{i}\right\}}^{m i n\left\{t-1, s_{i}\right\}} \xi_{i m s} \leq R_{b}^{\rho} \quad \forall b \in B ; \forall i \in I \\
\sum_{i=0}^{n} \sum_{m=1}^{M} r_{i m k}^{\nu} * \sum_{t=e s_{i}}^{l s_{i}} \xi_{i m t} \leq R_{k}^{\nu} \quad \forall k \in K \\
\xi_{i m t} \in\{0,1\}
\end{gathered}
$$

Kolisch and Sprecher [10] proposed a generator algorithm of instances for the uni-modal and multi-modal RCPSP (ProGen, http://www.om-db.wi.tum . $\mathrm{de} / \mathrm{psplib/main.html).} \mathrm{The} \mathrm{set} \mathrm{of} \mathrm{generated} \mathrm{instances} \mathrm{were} \mathrm{grouped} \mathrm{into} \mathrm{the}$ PSPLIB library. The purpose of this library is to provide a common set of test cases to evaluate the efficiency of newly developed methods to solve the RCPSP and the MRCPSP, and it has become a reference point for researchers of these problems. The test cases for the RCPSP consist of four sets $(j 30, j 60, j 90$, and $j 120$ ), each of which has 480 instances (except the set $j 120$ which has 600 instances), the test cases for the MRCPSP consist of seven sets $(j 10, j 12, j 14$,

Prepared using sagej.cls 
$j 16, j 18, j 20$, and $j 30$ ), each of which has 640 instances, with some of them being infeasible. For instances where the optimal solution is known, the average deviation regarding the optimal can be calculated. For those sets of problems whose optimal solution is unknown, the error is calculated based on the length of the critical path with the lowest execution time of activities, which is called the deviation to $L B 0$.

\section{MRCPSP-ENERGY: a MRCPSP extension for energy efficiency}

This paper focuses on the MRCPSP considering only renewable resources to propose MRCPSP-ENERGY, which incorporates energy consumption that allows the execution time of jobs to be changed. The analysis between energy consumption and makespan in scheduling is outlined below.

It assumes that energy is a resource consumed by activities. As usually occurs in the machinery of materials, the energy consumption of activities is independent of when the activities are scheduled [30]. Generally, the greater the energy consumption of an activity, the shorter its processing time. Typical examples are lathes, milling machines, rolling stock, elevators, etc. [13, 20]. Hence, here a similar energy behavior for MRCPSP activities is assumed. Finally, the total energy consumption of a project $\left(C E T P_{w}\right)$ can be calculated by Expression (7), where $e_{i m}$ is the energy consumption for activity $i$ executed in mode $m$.

$$
C E T P_{w}=\sum_{i=1}^{n} e_{i m}, m \in\{1,2, . ., M\}
$$

\section{Project efficiency: optimization criterion in MRCPSP-ENERGY}

In this section, an efficiency-based criterion is proposed. MRCPSP-ENERGY requires a bi-objective criterion that simultaneously minimizes both makespan (as the usual MRCPSP criterion) and energy consumption. In literature, when managing two objectives, a convex combination is normally used to generate the Pareto front; however, it is not appropriate for making a fair performance comparison between them since the weights of both objectives may vary from one method to another. Grouping them into a single objective that is related to the concept of efficiency allows us to obtain a better solution for the whole system without influencing the significance of the objectives.

Prepared using sagej.cls 
Generally, the concept of efficiency can be defined as the ratio between the energy supplied to a process and the transformed energy that it delivers. For example, in manufacturing machine operations (e.g., like milling, turning, drilling, etc.), the theoretical efficiency of an electric motor is the conversion of electrical energy into mechanical energy. This is represented by Expression (8), where $\eta$ is the efficiency, $P_{\text {mechanical }}$ represents the energy output from the motor shaft and, $P_{\text {electrical }}$ is the energy absorbed [31].

$$
\eta=\frac{P_{\text {mechanical }}}{P_{\text {electrical }}}=\frac{P_{\text {mechanical }}}{P_{\text {mechanical }}+\sum \text { losses }}
$$

Expression (8) is a theoretical efficiency, which cannot reach a value 1 because, during the transformation of electricity to mechanical energy, there are always losses, mostly in the form of heat. These losses tend to increase as the electrical energy consumption increases [32]. Therefore, efficiency is characterized by an initial increasing curve until it reaches a horizontal asymptote (see Figure 1-a). Different efficiency curves of different processes or machines maintain the same trend, varying according to the technical specifications of each machine. The theoretical behavior of efficiency is different from the real one since the real behavior of efficiency decreases after reaching a maximum. For instance, Figure 1-b shows the efficiency performance of a real $4 k w$ electric motor [31].
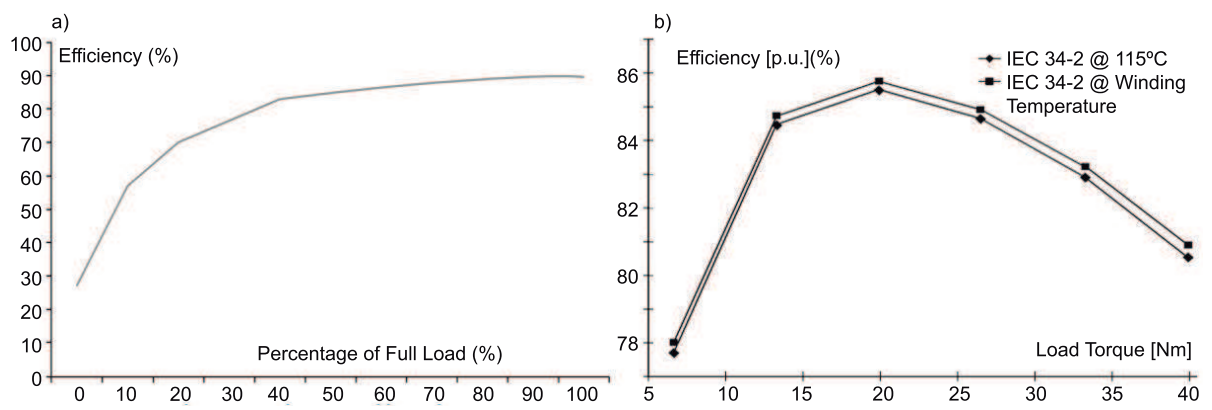

Figure 1. a) Theoretical efficiency of an engine [33]. b) Real efficiency of a motor [31].

When energy is supplied to a motor, the output is mechanical energy. Meanwhile, the output for a project in which activities are carried out by machines or people should be a duration that is affected by the amount of energy. The more energy, the shorter the duration until the highest reduction is obtained, and then more input might cause the machines to work slower due to overload, as occurs in Figure (1.b). Expression (9) describes this behavior for 
the duration $d_{i}$ and the energy consumption $e_{i}$ of an activity, but the range is not necessarily between 0 and 1 .

$$
\eta_{i}^{\prime}\left(e_{i}\right)=\frac{1 / d_{i}\left(e_{i}\right)}{e_{i}}
$$

The previous concept can be extended to an entire project, Expression (10). Nonetheless, as this relation is outside of the interval $[0-1]$, it cannot yet be considered an efficiency value. If the optimal values of makespan and energy consumption were known, Expression (10) could be standardized, but these values are unknown. Therefore, they are approximated by two lower bounds: $e^{e m i n} n_{w}$ and $L B 0 \mathrm{~min}_{w}$, respectively. The first is computed as $\left(\sum_{i=1}^{n} e_{i 1}\right)$ where $e_{i 1}$ is the minimum energy consumption of activity $i$. The second is estimated by using the critical path method, considering the minimum processing time of each activity $i$ within its $m$ modes. In this way, an upper bound of the project performance $C S R_{w}$ can be calculated to standardize Expression (10). Expression (11) shows how to calculate $C S R_{w}$. It can be interpreted as the ideal efficiency of a project.

$$
\eta_{w}^{*}=\frac{1 / \operatorname{makespan}_{w}}{C E T P_{w}}
$$

Expression (12) standardizes Expression (10) by dividing it by Expression (11). It is defined as the relative efficiency of the project $w$ with respect to an ideal efficiency value.

$$
\begin{gathered}
C S R_{w}=\frac{1 / \operatorname{LBOmin}_{w}}{\operatorname{emin}_{w}} \\
\eta_{w}\left(\operatorname{makespan}_{w}, C E T P_{w}\right)=\frac{1}{C S R_{w}} * \frac{1 / \operatorname{makespan}_{w}}{C E T P_{w}}
\end{gathered}
$$

As pointed out above, the energy consumption of a project is the sum of the energy consumption of its activities. However, it is important to remark that project efficiency is not equal to the sum of the efficiency of its activities. The reason is that the makespan of a project (which represents the inverse of $P_{\text {mechanical }}$ of the project) is not the sum of the durations of its activities.

On the basis of the above concepts, MRCPSP-ENERGY can be defined as follows:

Definition 1: MRCPSP-ENERGY is a project that consists of a set of $n$ activities $I=\{1, \ldots, i, \ldots, n\}$, a set $B$ of $K^{\rho}$ shared renewable resources $B=$

Prepared using sagej.cls 
$\left\{1, \ldots b, \ldots K^{\rho}\right\}$, and there is an available amount $R_{b}^{\rho}$ of every renewable resource. Each activity $i$ has $m=\{1, \ldots, M\}$ execution modes and a non-preemptive execution time $d_{i m}$, requiring a total of $r_{i b}$ renewable resources of type $b$ and an amount of energy $e_{i m}$ for its realization. Activities are subject to precedence constraints, which indicate that each activity cannot be started before all its predecessor activities are completed. The different energy consumptions for an activity give rise to different execution modes. Thus, MRCPSP-ENERGY is similar to the RCPSP, where the activities have different execution modes that are associated to different energy consumptions.

The main differences with respect to the MRCPSP are the following (1) the modes depend entirely on energy consumption $e_{i m} ;(2)$ the relation between energy consumption and processing time is inverse; and (3) the objective is to maximize the project efficiency, which means to minimize both energy consumption and makespan.

The formal mathematical model of MRCPSP-ENERGY maintains the same constraints as the MRCPSP, (constraints (2) to (6)) without the non-renewable resources constraints (5). The objective function for PSPLIB-ENERGY is reformulated according to Expression (13).

$$
\text { Max }: \eta_{w}\left(\text { makespan }_{w}, C E T P_{w}\right)
$$

\section{PSPLIB-ENERGY: An extension of the PSPLIB library}

In this section, the PSPLIB-ENERGY library is proposed. It complements PSPLIB with different levels of energy consumption and processing times associated to each activity. This extension aims to evaluate search methods for optimizing MRCPSP-ENERGY, taking into account both makespan and energy consumption, by using the proposed efficiency-based criterion.

\section{The proposed model for energy consumption}

In order to expand the PSPLIB library, the values of energy consumption for activities and their corresponding processing times must be consistent with the behavior of real machines. For this purpose, a standard value of energy consumption $e_{i}$ and processing time $d_{i}$ for each activity is assigned a priori. Afterwards, a mathematical model relates the standard values with every mode of an activity to compute its value of energy consumption and processing time.

Prepared using sagej.cls 
In machining, there is no global mathematical model that describes all behaviors of energy consumptions of machine tools. This is due to the fact that energy consumption behavior depends on several technical factors. Nevertheless, the behavior is quite similar in most machines. The relationship between energy consumption and processing time in machining has a decreasing trend [30]. Thus, Expression (14) is proposed, where $t_{i}\left(c_{i}\right)$ is the proportion of processing time compared with the standard duration of activity $i$, and $c_{i}$ is the proportion of energy consumption compared with the standard consumption of activity $i$. Expression (14) has a decreasing trend in a way similar to the energetic behavior in machine tools. This function represents an approximation of the proposed efficiency model in the previous section. The values of the constants $A_{1}=$ 4.0704 and $A_{2}=2.5093$ center the function in $c_{i}=1(100 \%)$ and $t_{i}=1(100 \%)$, representing the standard of the energy consumption and the processing time, respectively. As an example, a value of $c_{i}=1,6(160 \%)$ represents a consumption of $60 \%$ of additional energy for activity $i$, and a value obtained $t_{i}=0,67(67 \%)$ represents a decrease of $33 \%$ in the processing time of activity $i$. Figure 2 shows the graph of Expression (14).

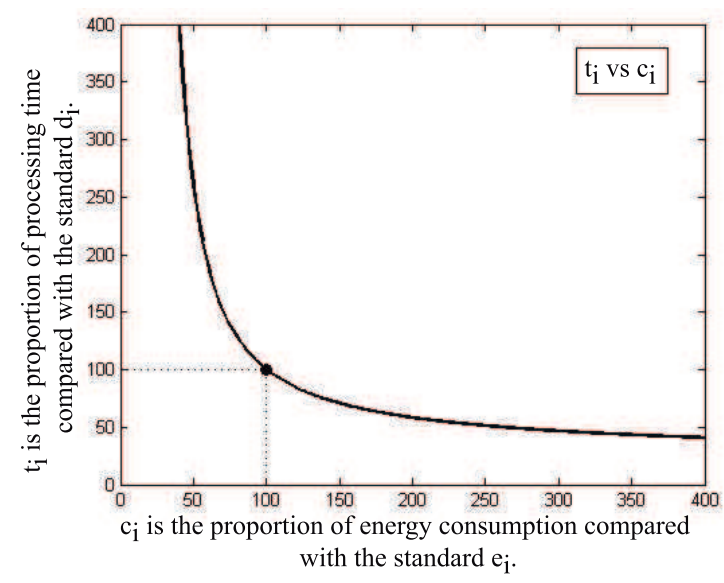

Figure 2. The proportion of processing time in relation to proportion of energy consumption, Expression (14).

$$
t_{i}\left(c_{i}\right)=\frac{A_{1} * \ln (2)}{\ln \left(1+\left(c_{i} * A_{2}\right)^{3}\right)}
$$

As a result of the above energy-time model, Expression $\eta_{i}\left(c_{i}\right)(15)$ is defined as the relative efficiency of activity $i$ depending on $c_{i}$. This expression can be 
interpreted as the efficiency percentage of activity $i$ respect to its efficiency, when both standard energy and standard processing time are used. For instance, a $\eta_{i}\left(c_{i}\right)=130 \%$ indicates that, given a proportion of energy consumption $c_{i}$, an efficiency of $30 \%$ higher than the standard is obtained. Figure 3 shows the relative efficiency Expression (15). It is important to note how the trend of the curve is similar to the real efficiency shown in $[34,31]$.

$$
\eta_{i}\left(c_{i}\right)=\frac{1 / t_{i}\left(c_{i}\right)}{c_{i}}=\frac{\ln \left(1+\left(c_{i} * A_{2}\right)^{3}\right)}{c_{i} * A_{1} * \ln (2)}
$$

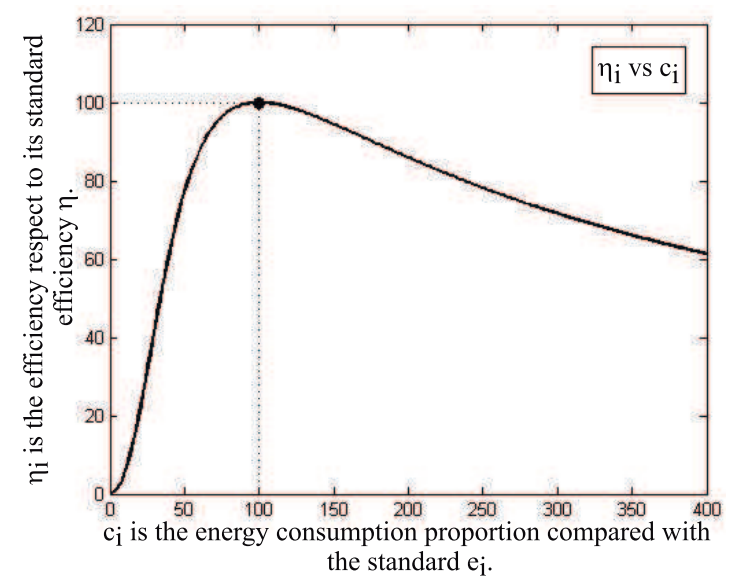

Figure 3. Relative efficiency of the activity, Expression (15).

\section{Energy extension for PSPLIB}

To extend PSPLIB to MRCPSP-ENERGY, energy consumption and processing times are included for each activity in the uni-modal RCPSP instances, through the mathematical model described above (Expression (14)). Therefore, the four problems sets $j 30, j 60, j 90$, and $j 120$ are created with $30,60,90$, and 120 activities, respectively. The values of the standard efficiency of the projects is available in the ENERGY-PSPLIB library.

Without loss of generality, three specific values for energy consumption $e_{i m}$ are taken. Therefore, three modes are defined. The first mode $\left(c_{i 1}=0.8\right)$ corresponds to a decrease of $20 \%$ in the energy consumption related to the standard value $e_{i 2}$. The second mode $\left(c_{i 2}=1\right)$ has the standard value of energy consumption $e_{i}^{\text {std }}=e_{i 2}$, which corresponds to the standard duration $d_{i}^{\text {std }}=d_{i 2}$

Prepared using sagej.cls 
provided by the original PSPLIB library. The third mode $\left(c_{i 1}=1.2\right)$ corresponds to an increase of $20 \%$ in energy consumption related to the standard value $e_{i 2}$.

The corresponding values of processing time $\left(d_{i m}\right)$ and energy consumption $\left(e_{i m}\right)$ can be calculated using Expressions (16) and (17), respectively. The values of $t_{i}\left(c_{i m}\right)$ are calculated using Expression (14).

$$
\begin{gathered}
d_{i m}=t_{i}\left(c_{i m}\right) * d_{i}^{s t d}, \quad i \in I, m \in\{1,2,3\} \\
e_{i m}=c_{i m} * e_{i}^{s d t}, \quad i \in I, m \in\{1,2,3\}
\end{gathered}
$$

The values of $e_{i}^{s d t}$ are defined by assigning a random consumption value with an interval $[1,10]$ to each instance's activity on the PSPLIB-ENERGY library. This range of values was proposed considering the same intervals used for the original parameters in the PSPLIB library. Since all parameter values in the PSPLIB library are integers, the values of the parameters for the PSPLIBENERGY library are also integers. Note that an activity $i$ with duration $d_{i m}=1$ cannot be reduced. Similarly, if an activity $i$ has an energy consumption of $e_{i m}=1$, it cannot be reduced. The approximation of the other cases were made taking into account their corresponding mode $m$. Then, for $m=3, d_{i m}$ was rounded downwards and $e_{i m}$ was rounded upwards. In the case $m=1, d_{i m}$ was rounded upwards and $e_{i m}$ was rounded downwards.

For the evaluation of future techniques to be developed using the PSPLIBENERGY library, the evaluation criteria is to maximize the relative efficiency presented in Expression (12). This expression takes into account both the makespan $_{w}$ and the total energy consumption $C E T P_{w}$ (Expression (7)) in a single-objective.

To calculate the average value $\bar{\eta}$ for the problems of the sets $j 30, j 60, j 90$, or $j 120$ in PSPLIB-ENERGY, Expression (18) is defined, where $n P$ is the number of evaluated projects.

$$
\bar{\eta}=\frac{\sum_{w=1}^{n P} \eta_{w}\left(\operatorname{makespan}_{w}, C E T P_{w}\right)}{n P}
$$

\section{Example of a MRCPSP-ENERGY instance}

In this section, an example of a small MRCPSP-ENERGY instance is presented. This instance consists of $n=8$ activities and $B=3$ renewable resources. Table 1 shows the energy consumption $\left(e_{i m}\right)$, the processing time $\left(d_{i m}\right)$, and the

Prepared using sagej.cls 
resource usage. The first column shows the activities (activities 1 and 8 are fictitious with duration and consumption equal to 0 ). The second to the seventh columns show the modified duration of the activities and the corresponding energy consumption $\left(d_{i m}, e_{i m}\right)$. The values of $d_{i 2}$ and $e_{i 2}$ (columns in bold) are considered the standard values with a usage of $100 \%$ in energy consumption and processing time. The eighth to the tenth columns show the resource usage.

Table 1. Energy consumption $\left(e_{i m}\right)$, processing time $\left(d_{i m}\right)$, and resource usage for the MRCPSP-ENERGY instance.

\begin{tabular}{c|cc|cc|cc|ccc}
\hline \hline Num.Job & $d_{i 1}$ & $e_{i 1}$ & $d_{i 2}$ & $e_{i 2}$ & $d_{i 3}$ & $e_{i 3}$ & $r_{i 1}$ & $r_{i 2}$ & $r_{i 3}$ \\
\hline 1 & 0 & 0 & $\mathbf{0}$ & $\mathbf{0}$ & 0 & 0 & 0 & 0 & 0 \\
2 & 4 & 4 & $\mathbf{3}$ & $\mathbf{5}$ & 2 & 6 & 1 & 1 & 1 \\
3 & 3 & 1 & $\mathbf{2}$ & $\mathbf{2}$ & 1 & 3 & 2 & 1 & 1 \\
4 & 2 & 5 & $\mathbf{1}$ & $\mathbf{7}$ & 1 & 7 & 1 & 0 & 1 \\
5 & 7 & 3 & $\mathbf{5}$ & $\mathbf{4}$ & 4 & 5 & 0 & 1 & 1 \\
6 & 3 & 4 & $\mathbf{2}$ & $\mathbf{6}$ & 1 & 8 & 2 & 1 & 0 \\
7 & 8 & 4 & $\mathbf{6}$ & $\mathbf{6}$ & 5 & 8 & 2 & 1 & 2 \\
8 & 0 & 0 & $\mathbf{0}$ & $\mathbf{0}$ & 0 & 0 & 0 & 0 & 0 \\
\hline
\end{tabular}

Figure 4 shows the precedence relationships and the information provided in Table 1: the use of each renewable resource, and the processing time versus the energy consumption in each mode of execution.

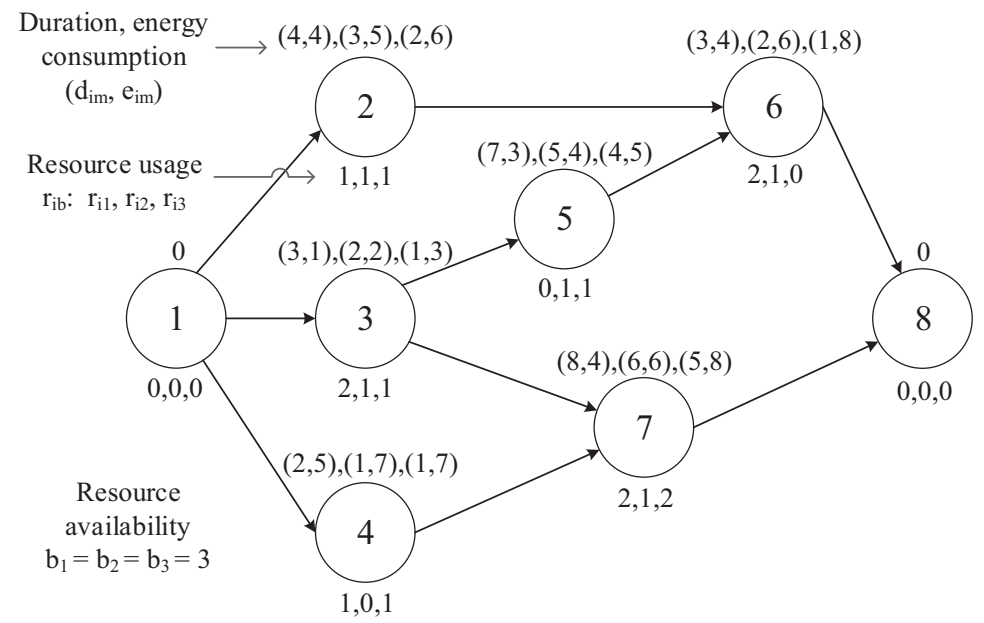

Figure 4. A network for the MRCPSP-ENERGY instance.

Figure 5 shows three Gantt charts of three solutions for the given instance. Figure 5.a shows an optimal solution when the problem only uses a proportion

Prepared using sagej.cls 
of energy consumption $c_{i}=100 \%$. Figure 5.b and Figure 5.c represent two solutions using different energy consumptions, and, consequently, different processing times.

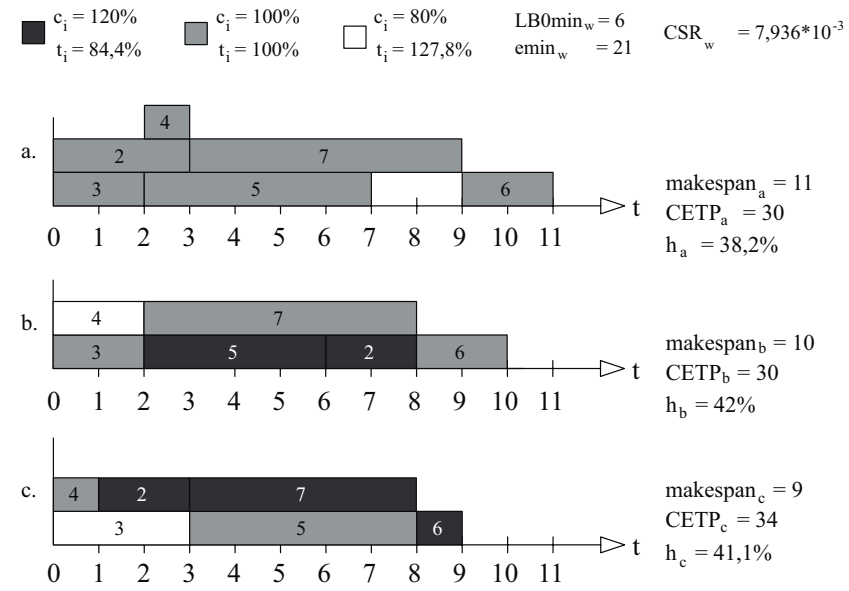

Figure 5. Three Gantt charts of the example in Figure 4.

To calculate the efficiency $\eta_{w}$ of each solution, the values $L B 0_{w}=6$ and $\operatorname{emin}_{w}=21$ were calculated from Figure 4 . Figure 5 shows the upper level of project performance $C S R_{w}$, the value of the total project duration makespan $_{w}$, the total energy consumption of the project $C E T P_{w}$, and the value of the evaluation criterion $\eta_{w}$ of three different solutions.

Solution (a) has the lowest value of efficiency $\eta_{a}=38,2 \%$. The lowest makespan is achieved in Solution $(c)$. However, it has the highest energy consumption, its efficiency is $\eta_{c}=41,1 \%$, and it is considered better than Solution (a). Solution (b) maintains the same energy consumption as Solution (a) but improves in makespan. Therefore, it achieves the highest value of the relative efficiency of the project $\eta_{b}=42 \%$. In conclusion, Solution $(b)$ is considered to be the best solution of the three.

It is noteworthy that the efficiency values are relatively low in all cases (close to $40 \%$ ) because they are compared with the ideal value of the upper bound of project performance $C S R_{w}$.

Prepared using sagej.cls 


\section{A genetic algorithm for solving the PSPLIB-ENERGY library}

Generally, genetic algorithms are well-recognized and appropriate approaches for solving MRCPSP [27, 35]. Therefore, in this section, a genetic algorithm is proposed for solving MRCPSP-ENERGY library instances. This is considered a good starting point for the research community to compare the performance of their techniques for solving MRCPSP-ENERGY library instances. The main contribution in this section is the new application and adaptation of a genetic algorithm to the proposed library. The pseudo code for the proposed genetic algorithm is shown in Algorithm 1.

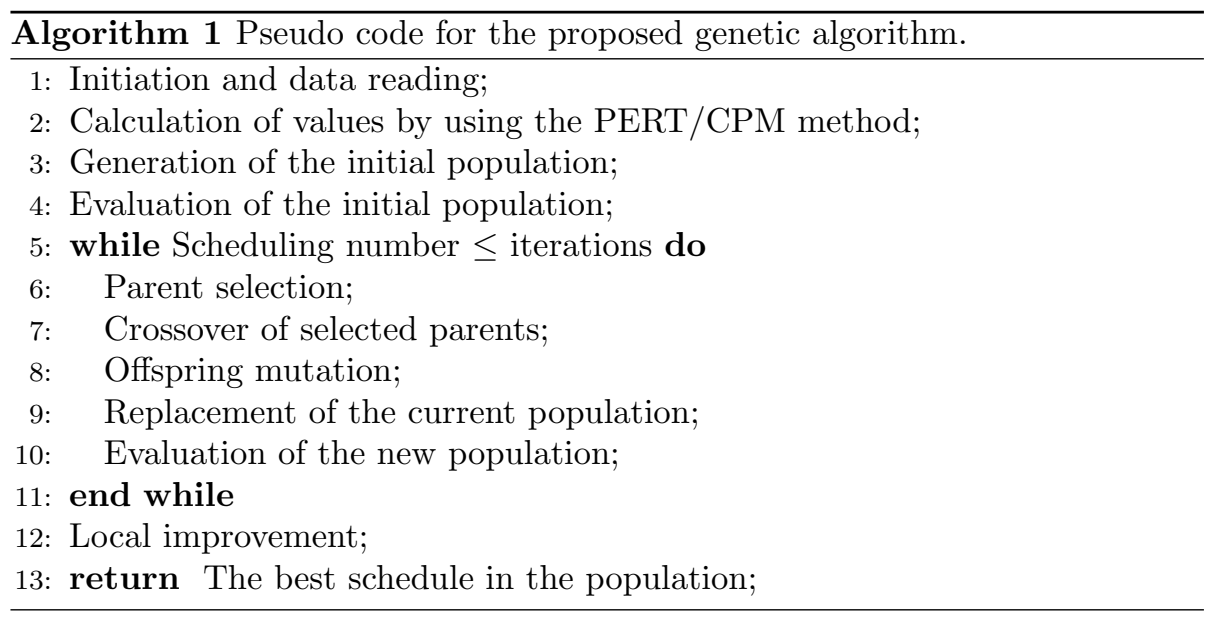

Codification of solutions. There are several compatible codifications for modeling the MRCPSP in terms of genes and chromosomes [36]. The activity list representation and random key representation are those that have obtained the best results [27]. In this work, the activity list is used as codification. It consists of a precedence feasible activity list $\left\{a_{1}, a_{2}, \ldots, a_{i}, \ldots, a_{n}\right\}$, in which the activity position in the list represents the priority to be scheduled.

A chromosome is created to contain all of the information of a complete feasible schedule. It consists of $n$ activity genes (the activity list), one schedule generation scheme (SGS) gene, and one direction gene. The activity genes have a pair of values $\left(a_{i}, z_{i}\right)$, where $a_{i}$ represents the activity position and $z_{i}$ represents how the activity consumes energy. The SGS gene is 0 when the serial scheme is used and 1 when the parallel scheme is used. The direction gene is 0 when the forward direction is used and 1 when the backward direction is used. An example of the codification used is shown in Figure 6.

Prepared using sagej.cls 


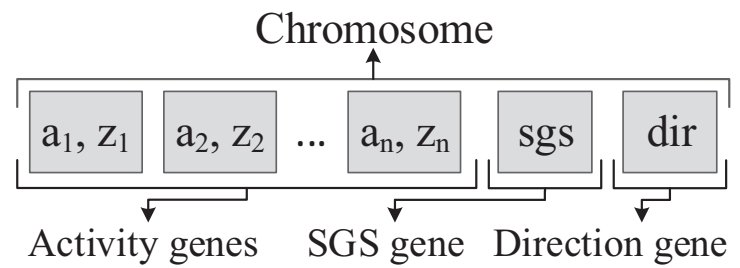

Figure 6. The codification used in the genetic algorithm.

The initial population and population size. Following Kolish et al. [36], the regret based biased random sampling (RBBRS) and the latest start time (LST) priority rule [37, 38] are used to generate an initial population, with the parameters $\epsilon=1$ and $\alpha=1$.

In the methodologies for solving the MRCPSP, there is no standard method to estimate an appropriate population size. Debels and Vanhoucke [39] state that the larger the number of activities, the smaller the population size. Cervantes et al. [40] propose a mathematical expression where the relation between the population and the activities is inverse. Based on these statements, Expression (19) and Expression (20) are proposed.

$$
\begin{gathered}
\text { population }=\left(\frac{2700}{N}\right) * \ln \left(\frac{e * \text { iterations }}{1000}\right) \text { with } N \leq 120 \\
\text { generations }=\frac{\text { iterations }}{\text { population }}
\end{gathered}
$$

Fitness function. The fitness function allows the quality of solutions to be determined. The proposed relative efficiency (Expression (12)) is used in this work as a fitness function. Thus, a solution with a greater relative efficiency value $\eta_{w}$ is considered better than another with a lower value.

Selection. In the MRCPSP, roulette and ranking are the most commonly used selection methods. The selection by roulette does not work properly when the range between the fitness values of individuals gets bigger. It happens in the MRCPSP, but not in MRCPSP-ENERGY because all of the values of relative efficiency of the project are ranged in the interval $[0,1]$. Therefore, the roulette selection is used, in which the parents are selected according to their fitness. The best chromosomes are more likely to be selected [41].

Crossover. An appropriate crossover operator has to be defined for each gene type. For activity genes, one-point crossover is used. A random integer $q$

Prepared using sagej.cls 
with $0<q<N$ is generated. Thus, the first genes from 0 to $q$ are taken from Parent 1 while the remaining ones are taken from Parent 2 . Thus, the list of activity genes is gone over one by one and only those that are different from Parent 1 are chosen. For SGS and direction genes, the gene value is inherited when it is the same in both parents, otherwise it is randomly generated.

Mutation. The proposed genetic algorithm uses the insertion of Boctor [42] because it is considered a good operator since it produces feasible solutions and is compatible with the activity list codification. Thus, given an activity $i$ to be inserted into the activity list, first, the maximum position of all its predecessors in the list (maxPred) and the minimum position of all its successors ( $\operatorname{minSuc}$ ) are computed, and then a random integer $R$ nd is generated with maxPred $\leq$ Rnd $\leq$ minSuc. Activity $i$ is inserted in this position. A 0.05 mutation probability value for each activity is selected. An activity that has been selected to mutate will change the position in the activity list as well as its energy consumption and execution time.

Replacement. The replacement strategies are the way the next generations are formed. A semi-elitist strategy is used, which consists of building the new population from the individual with the best solution in the current population and the offspring of that population.

Local improvement. Finally, a local improvement is applied to the best solution. It consists of going over all of the activities and checking if they can be executed with less energy consumption (longer execution time) without breaking precedence or resource constraints.

\section{Evaluation and analysis of results}

The experiments were performed on a PC with Intel(R) Core(TM) i7 CPU to $2.20 \mathrm{GHz}$ and $16 \mathrm{gb} \mathrm{ram}$. The proposed genetic algorithm was developed in $C++$. First, the results are shown by keeping the standard academic format, then the behavior of the proposed algorithm is presented. Finally, how different energy levels can increase process efficiency is analyzed.

The results are shown in Table 2. The sets of problems $j \#$ are shown in the first column and the remaining columns show the average value of relative efficiency $(\bar{\eta})$ for the problem sets $j 30, j 60, j 90$, and $j 120$ of the PSPLIBENERGY library, for 1,000, 5,000, and 50,000, respectively. In [27], Kolisch and Hartmann clarify the reason for using iterations as comparison criterion,

Prepared using sagej.cls 
and they define what an iteration is. In Table 3 the execution times (in seconds) are shown for the same number of iterations (1,000, 5, 000 and 50,000).

Table 2. $\bar{\eta}$ obtained by using the proposed genetic algorithm for solving the MRCPSP-ENERGY library.

\begin{tabular}{cccc}
\hline \hline $\mathrm{j} \#$ & \multicolumn{3}{c}{ Iterations $/ \bar{\eta}$} \\
& 1,000 & 5,000 & 50,000 \\
\hline $\mathrm{j} 30$ & 0.5966 & 0.6091 & 0.6293 \\
$\mathrm{j} 60$ & 0.6029 & 0.6182 & 0.6424 \\
$\mathrm{j} 90$ & 0.6052 & 0.6216 & 0.6478 \\
$\mathrm{j} 120$ & 0.4760 & 0.4875 & 0.5032 \\
\hline
\end{tabular}

Table 3. Processing time obtained by using the proposed genetic algorithm for solving the MRCPSP-ENERGY library.

\begin{tabular}{cccc}
\hline \hline $\mathrm{j} \#$ & \multicolumn{3}{c}{ Iterations/seconds } \\
& 1,000 & 5,000 & 50,000 \\
\hline $\mathrm{j} 30$ & $0.053 s$ & $0.198 s$ & $1.514 s$ \\
$\mathrm{j} 60$ & $0.159 s$ & $0.544 s$ & $4.163 s$ \\
$\mathrm{j} 90$ & $0.286 s$ & $0.957 s$ & $8.210 s$ \\
$\mathrm{j} 120$ & $0.424 s$ & $1.282 s$ & $10.624 s$ \\
\hline
\end{tabular}

To test the consistency and the convergence behavior of the proposed genetic algorithm, the algorithm was executed up to 100,000 iterations. The relative efficiency $(\bar{\eta})$ of each iteration for all sets is shown in Figure 7 . It exhibits a typically growing function with a horizontal asymptote when the iterations tend toward infinity for the four sets. In fact, all of the sets obtained an average improvement that was lower than $0.083 \%$ in the last 5, 000 iterations.

On the other hand, it is important to analyze the search space when the energy consumption is included. In order to do this, the results obtained for solving PSPLIB-ENERGY using one and three levels of energy are compared (see Figure 8). For the first case, only the standard duration and the standard energy consumption (i.e., $d_{i 2}$ and $e_{i 2}$ ) are used, whereas, for the second case, the three levels of energy consumption are used. It can be observed that as the number of energy levels increases, a better solution can be achieved.

\section{Conclusions and further works}

Currently, many research works follow the strong trend to develop models that include an energy component for obtaining a sustainable solution to scheduling problems. As a result, it is imperative to develop new libraries to evaluate the behavior of new developed heuristics.

In this paper a efficiency-based MRCPSP is proposed. It is called MRCPSPENERGY, which includes energy consumption in a scheduling problem. The proposed bi-objective approach consists of maximizing relative project efficiency. This is carried out by simultaneously minimizing the makespan and the energy consumption. The efficiency concept tries to find the best solution for the system as a whole and to avoid the generation of the Pareto front. Furthermore, the

Prepared using sagej.cls 

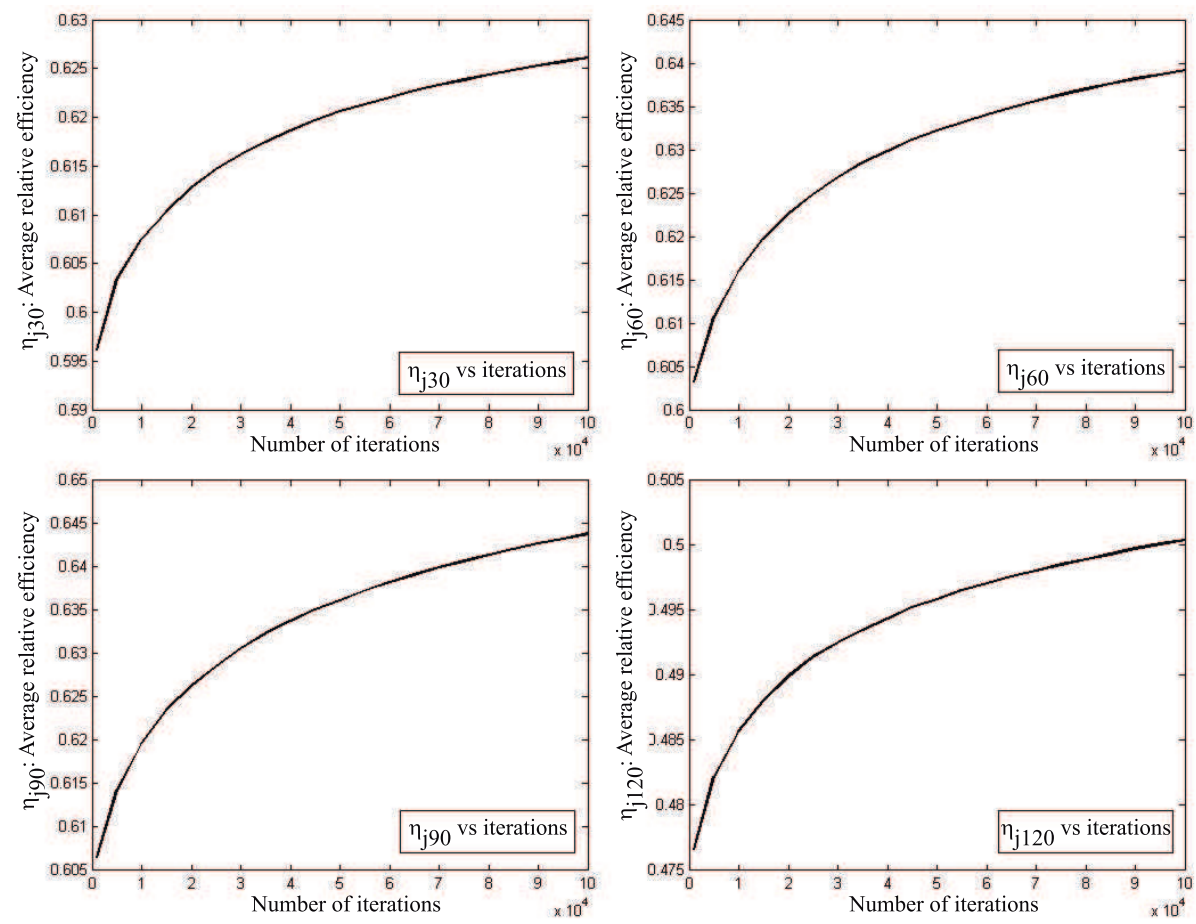

Figure 7. Convergence behavior of the proposed genetic algorithm for sets $j 30, j 60, j 90$, and $j 120$.

library PSPLIB-ENERGY is proposed. It is an extension to PSPLIB library for assessing the MRCPSP-ENERGY solution methods. This extension is supported by a proposed model of energy consumption. This model is consistent with the surveys reported in the academic literature about energy consumption for machines. Therefore, this library can be used to evaluate and compare different solving methods.

Since genetic algorithms have been competitive methods for solving the classical MRCPSP, a genetic algorithm has been proposed to solve MRCPSPENERGY by adapting well-known strategies that have been previously developed. The results show the convenience of assigning different energy consumptions and processing times to activities in machine scheduling problems in order to achieve energy-efficient solutions. The aim of these results is to establish an initial comparison point for future solving methods.

The PSPLIB-ENERGY library has been developed, keeping the same format as the PSPLIB library, with four sets of problems $(j 30, j 60, j 90$, and $j 120)$,

Prepared using sagej.cls 

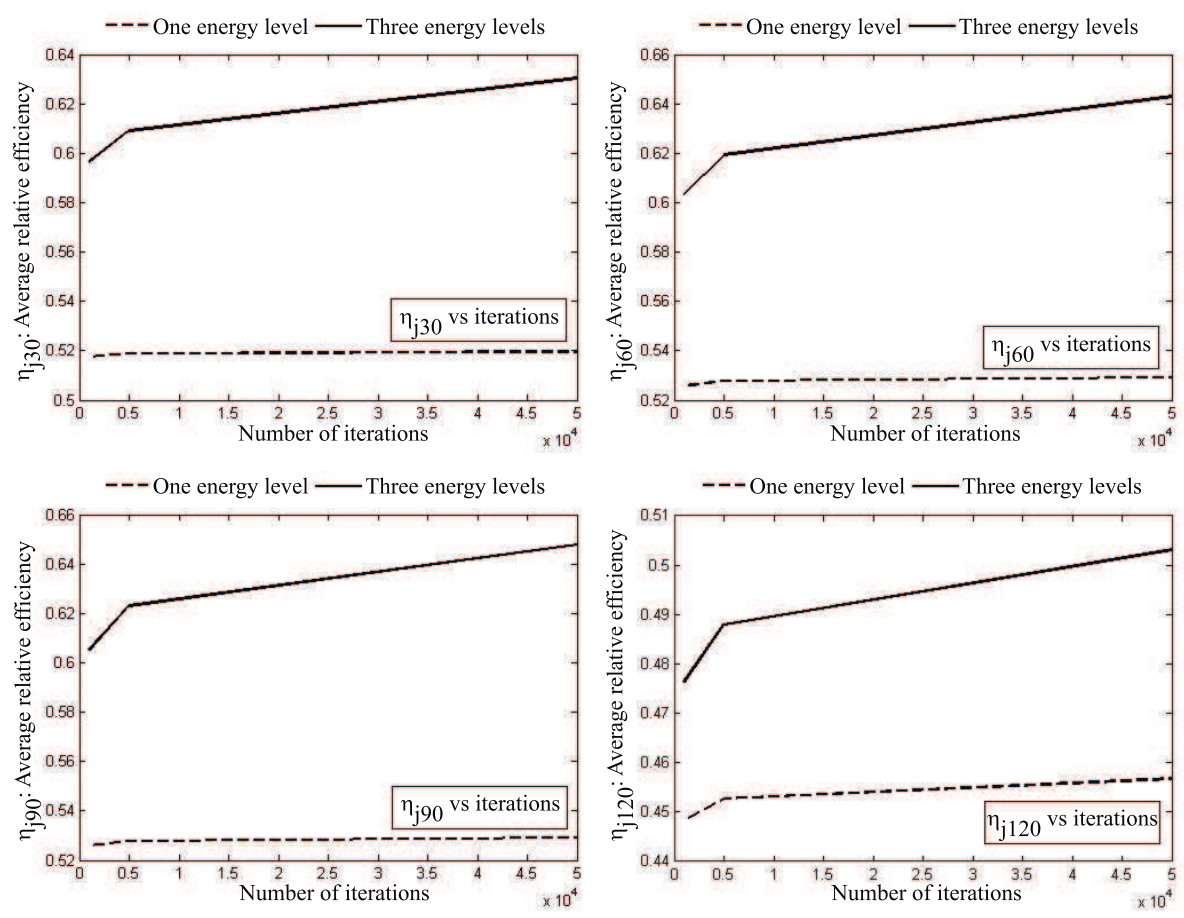

Figure 8. Comparison between levels of energy consumption and relative efficiency of sets $j 30, j 60, j 90$, and $j 120$.

each of which has 480 problems (except the set $j 120$, which has 600 instances). This library is available at http://gps.webs.upv.es/psplib-energy/.

In further works, we aim to extend this work to consider the energy consumption as a non-renewable resource with a dynamic threshold. This problem occurs when a limited energy budget is allocated to a project or the available energy is limited in some time intervals. As long as the available energy allows the construction of feasible solutions, the new solutions must take into account these dynamic thresholds, so the search must be focused on these bottlenecks to obtain energy efficient solutions. Thus, our metaheuristic proposal must be extended to address this issue.

Finally, this paper aims to encourage the development of future research to find sustainable and efficient solutions in resource-constrained scheduling problems.

Prepared using sagej.cls 


\section{Acknowledgements}

This research has been supported by the Spanish Government under the research projects TIN2013 - 46511-C2-1 and TIN2016-80856-R.

\section{References}

[1] Mouzon G, Yildirim MB and Twomey J. Operational methods for minimization of energy consumption of manufacturing equipment. International Journal of Production Research 2007; 45(18-19): 4247-4271.

[2] Rahimifard S, Seow Y and Childs T. Minimising Embodied Product Energy to support energy efficient manufacturing. CIRP Annals - Manufacturing Technology 2010; 59(1): 25-28.

[3] Cucala aP, Fernández a, Sicre C et al. Fuzzy optimal schedule of high speed train operation to minimize energy consumption with uncertain delays and drivers behavioral response. Engineering Applications of Artificial Intelligence 2012; 25(8): 1548-1557.

[4] Govindan K, Sarkis J, Jabbour CJC et al. Eco-efficiency based green supply chain management: Current status and opportunities. European Journal of Operational Research 2014; 233(2): 293-298.

[5] Hassini E, Surti C and Searcy C. A literature review and a case study of sustainable supply chains with a focus on metrics. International Journal of Production Economics 2012; 140(1): 69-82.

[6] Escamilla J and Salido MA. A dual scheduling model for optimizing robustness and energy consumption in manufacturing systems. Proceedings of the Institution of Mechanical Engineers, Part B: Journal of Engineering Manufacture 2016; : 0954405415625915.

[7] Zhang C, Jiang P, Zhang L et al. Energy-aware integration of process planning and scheduling of advanced machining workshop. Proceedings of the Institution of Mechanical Engineers, Part B: Journal of Engineering Manufacture 2016; : 0954405415616785.

[8] Iqbal N, Aziz MH, Jahanzaib M et al. Integration of cell formation and job sequencing to minimize energy consumption with minimum make-span. Proceedings of the Institution of Mechanical Engineers, Part B: Journal of Engineering Manufacture 2016; : 0954405416630182.

Prepared using sagej.cls 
[9] Lova A, Tormos P, Cervantes M et al. An efficient hybrid genetic algorithm for scheduling projects with resource constraints and multiple execution modes. International Journal of Production Economics 2009; 117(2): 302316.

[10] Kolisch R and Sprecher A. PSPLIB - A project scheduling library. European Journal of Operational Research 1996; 96: 205-216.

[11] Salonitis K and Ball P. Energy Efficient Manufacturing from Machine Tools to Manufacturing Systems. Procedia CIRP 2013; 7: 634-639.

[12] Seow Y and Rahimifard S. A framework for modelling energy consumption within manufacturing systems. CIRP Journal of Manufacturing Science and Technology 2011; 4(3): 258-264.

[13] Li L, Yan J and Xing Z. Energy requirements evaluation of milling machines based on thermalequilibrium and empirical modelling. Journal of Cleaner Production 2013; 52: 113-121.

[14] Wu C, Li B and Liang SY. A critical energy model for brittle-ductile transition in grinding considering wheel speed and chip thickness effects. Proceedings of the Institution of Mechanical Engineers, Part B: Journal of Engineering Manufacture 2016; : 0954405416654194.

[15] Moradnazhad M and Unver HO. Energy efficiency of machining operations: A review. Proceedings of the Institution of Mechanical Engineers, Part B: Journal of Engineering Manufacture 2016; : 0954405415619345.

[16] Bruzzone A, Anghinolfi D, Paolucci M et al. Energy-aware scheduling for improving manufacturing process sustainability: A mathematical model for flexible flow shops. CIRP Annals - Manufacturing Technology 2012; 61(1): 459-462.

[17] Niu S, Ong S and Nee A. An improved intelligent water drops algorithm for solving multi-objective job shop scheduling. Engineering Applications of Artificial Intelligence 2013; 26(10): 2431-2442.

[18] He Y and Liu F. Methods for Integrating Energy Consumption and Environmental Impact Considerations into the Production Operation of Machining Processes. Chinese Journal of Mechanical Engineering 2010; 23.

Prepared using sagej.cls 
[19] Duflou JR, Sutherland JW, Dornfeld D et al. Towards energy and resource efficient manufacturing: A processes and systems approach. CIRP Annals - Manufacturing Technology 2012; 61(2): 587-609.

[20] Fang K, Uhan N, Zhao F et al. A new approach to scheduling in manufacturing for power consumption and carbon footprint reduction. Journal of Manufacturing Systems 2011; 30(4): 234-240.

[21] Artigues C, Lopez P and Haït A. The energy scheduling problem: Industrial case-study and constraint propagation techniques. International Journal of Production Economics 2013; 143(1): 13-23.

[22] Luo H, Du B, Huang GQ et al. Hybrid flow shop scheduling considering machine electricity consumption cost. International Journal of Production Economics 2013; 146(2): 423-439.

[23] Okubo H, Miyamoto T, Yoshida S et al. Project scheduling under partially renewable resources and resource consumption during setup operations. Computers $\mathscr{E}$ Industrial Engineering 2015; 83: 91-99.

[24] Zhang Z, Tang R, Peng T et al. A method for minimizing the energy consumption of machining system: integration of process planning and scheduling. Journal of Cleaner Production 2016; .

[25] Li S, Liu F and Zhou X. Multi-objective energy-saving scheduling for a permutation flow line. Proceedings of the Institution of Mechanical Engineers, Part B: Journal of Engineering Manufacture 2016; : 0954405416657583.

[26] Dai M, Tang D, Giret A et al. Energy-efficient scheduling for a flexible flow shop using an improved genetic-simulated annealing algorithm. Robotics and Computer-Integrated Manufacturing 2013; 29(5): 418-429.

[27] Kolisch R and Hartmann S. Experimental investigation of heuristics for resource-constrained project scheduling: An update. European Journal of Operational Research 2006; 174(1): 23-37.

[28] Talbot FB. Resource-Constrained Project Scheduling with Time-Resource Tradeoffs: The Nonpreemptive Case. Management Science 1982; 28(10): 1197-1210.

Prepared using sagej.cls 
[29] Blazewicz J, Lenstra J and Kan A. Scheduling subject to resource constraints: classification and complexity. Discrete Applied Mathematics $1983 ; 5(1): 11-24$.

[30] Oberg E, Jones FD, Horton HL et al. Machinery's Handbook. 27 ed. New York, USA: Industrial Press Inc., 2004. ISBN 0-8311-2737-6.

[31] Boglietti A, Cavagnino A, Lazzari M et al. International Standards for the Induction Motor Efficiency Evaluation: A Critical Analysis of the StrayLoad Loss Determination. IEEE Transactions on Industry Applications 2004; 40(5): 1294-1301.

[32] Saidur R. A review on electrical motors energy use and energy savings. Renewable and Sustainable Energy Reviews 2010; 14(3): 877-898.

[33] Beggs C. Energy: management, Supply and Conservation. Butterworth Heinemann, 2002. ISBN 0750650966.

[34] Draganescu F, Gheorghe M and Doicin C. Models of machine tool efficiency and specific consumed energy. Journal of Materials Processing Technology $2003 ; 141(1): 9-15$.

[35] Van Peteghem V and Vanhoucke M. An experimental investigation of metaheuristics for the multi-mode resource-constrained project scheduling problem on new dataset instances. European Journal of Operational Research 2014; 235(1): 62-72.

[36] Kolisch R and Hartmann S. Heuristic Algorithms for the ResourceConstrained Project Scheduling Problem: Classification and Computational Analysis. In Weglarz J (ed.) Project Scheduling, chapter 7. Springer US. ISBN 978-1-4615-5533-9, 1999. pp. 147-178.

[37] Hartmann S and Kolisch R. Experimental evaluation of state-of-theart heuristics for the resource-constrained project scheduling problem. European Journal of Operational Research 2000; 127(2): 394-407.

[38] Klein R. Bidirectional planning: improving priority rule-based heuristics for scheduling resource-constrained projects. European Journal of Operational Research 2000; 127(3): 619-638.

Prepared using sagej.cls 
[39] Debels D and Vanhoucke M. A Decomposition-Based Genetic Algorithm for the Resource-Constrained Project-Scheduling Problem. Operations Research 2007; 55(3): 457-469.

[40] Cervantes M, Lova A, Tormos P et al. A Dynamic Population SteadyState Genetic Algorithm for the Resource-Constrained Project Scheduling Problem. In Thanh Nguyen N, Borzemski L, Grzech A et al. (eds.) New Frontiers in Applied Artificial Intelligence, volume 502 ed. Springer Berlin Heidelberg. ISBN 978-3-540-69045-0, 2008. pp. pp 611-620.

[41] Hartmann S. A competitive genetic algorithm for resource-constrained project scheduling. Naval Research Logistics 1998; 45(7): 733-750.

[42] Boctor FF. Resource-constrained project scheduling by simulated annealing. International Journal of Production Research 1996; 34(8): 23352351.

Prepared using sagej.cls 


\section{Appendices}

\section{List of symbols}

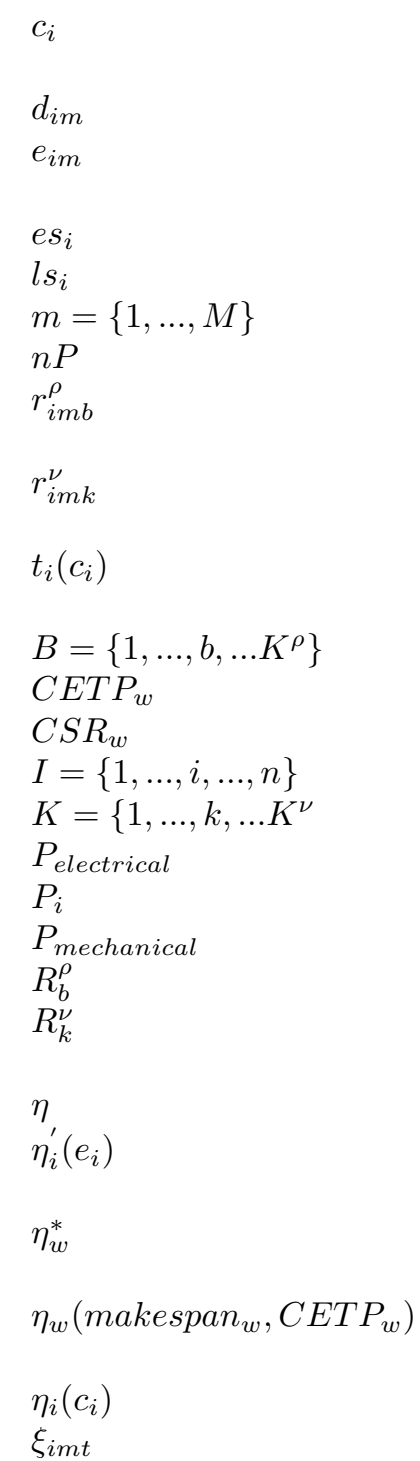

The proportion of energy consumption compared with the standard consumption of the activity $i$.

The duration of activity $i$ in execute mode $m$.

The energy consumption for activity $i$ executed in mode $m$.

The early start of activity $i$.

The late start of activity $i$.

A set of $M$ execution modes.

The total number of evaluated projects.

The amount of renewable resource of kind $b$ consumed by activity $i$ in execution mode $m$.

The amount of non-renewable resource of kind $k$ consumed by activity $i$ in execution mode $m$.

The proportion of processing time compared with the standard duration of activity $i$.

A set of $K^{\rho}$ shared renewable resources.

The total energy consumption of a Project $w$.

The upper bound of the project performance.

A set of $n$ activities.

A set of $K^{\nu}$ non-renewable resources.

The input energy.

A set of immediate predecessor activities of activity $i$.

The output energy.

The maximum amount of every renewable resource $b$.

The maximum amount of every non-renewable resource $k$.

The efficiency concept.

The relation between $d_{i}$ and $e_{i}$ of an activity $i$. The range is not necessarily between 0 and 1 .

The relation between makespan and CETP of a project $w$. The range is not necessarily between 0 and 1 .

The relative efficiency of a project $w$ with respect to $C S R_{w}$.

The relative efficiency of activity $i$ depending on $c_{i}$.

Binary decision variable, which takes a value of one when the activity $i$ is executed in mode $m$ and finishes at time $t$, and zero otherwise.

Prepared using sagej.cls 


\section{List of Tables}

1 Energy consumption $\left(e_{i m}\right)$, processing time $\left(d_{i m}\right)$, and resource usage for the MRCPSP-ENERGY instance. . . . . . . . . . . . . 14

$2 \bar{\eta}$ obtained by using the proposed genetic algorithm for solving the MRCPSP-ENERGY library. . . . . . . . . . . . . . . . . . 19

3 Processing time obtained by using the proposed genetic algorithm for solving the MRCPSP-ENERGY library. . . . . . . . . . . . . 19

\section{List of Figures}

1 a) Theoretical efficiency of an engine [33]. b) Real efficiency of a motor $[31] \ldots \ldots \ldots \ldots \ldots \ldots$

2 The proportion of processing time in relation to proportion of energy consumption, Expression (14). . . . . . . . . . . 11

3 Relative efficiency of the activity, Expression (15). . . . . . . 12

4 A network for the MRCPSP-ENERGY instance. . . . . . . . . . 14

5 Three Gantt charts of the example in Figure 4. . . . . . . . . . 15

6 The codification used in the genetic algorithm. . . . . . . . 17

7 Convergence behavior of the proposed genetic algorithm for sets $j 30, j 60, j 90$, and $j 120 \ldots \ldots \ldots \ldots \ldots \ldots \ldots \ldots$

8 Comparison between levels of energy consumption and relative efficiency of sets $j 30, j 60, j 90$, and $j 120 \ldots \ldots \ldots \ldots \ldots$ 